\title{
Pressure Effects on Protein Hydration Water Thermodynamics
}

\section{Supplementary Material}

\author{
Viren Pattni and Matthias Heyden* \\ School of Molecular Sciences, Arizona State University, Tempe, AZ 85287, U.S.A. \\ E-mail: mheyden1@asu.edu
}




\section{Spatially resolved thermodynamic properties of water}
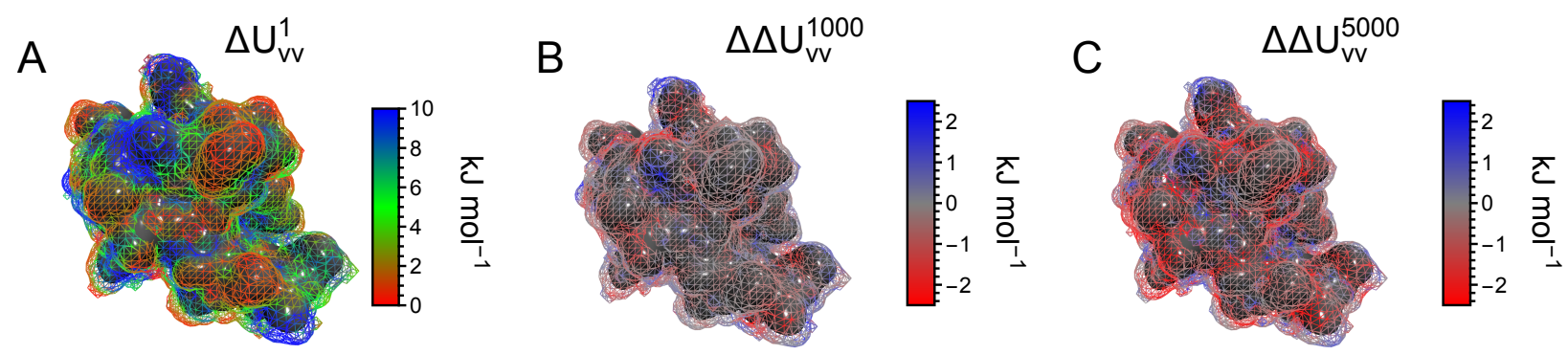

Figure S1: Three-dimensional representation of properties of water molecules in the protein hydration shell at $3 \AA$ from the protein surface: (A) change in water-water interaction energies per molecule from bulk at 1 bar, $\Delta U_{\mathrm{vv}}$; (B)-(C) deviations of $\Delta U_{\mathrm{vv}}$ at the corresponding pressures from 1 bar.
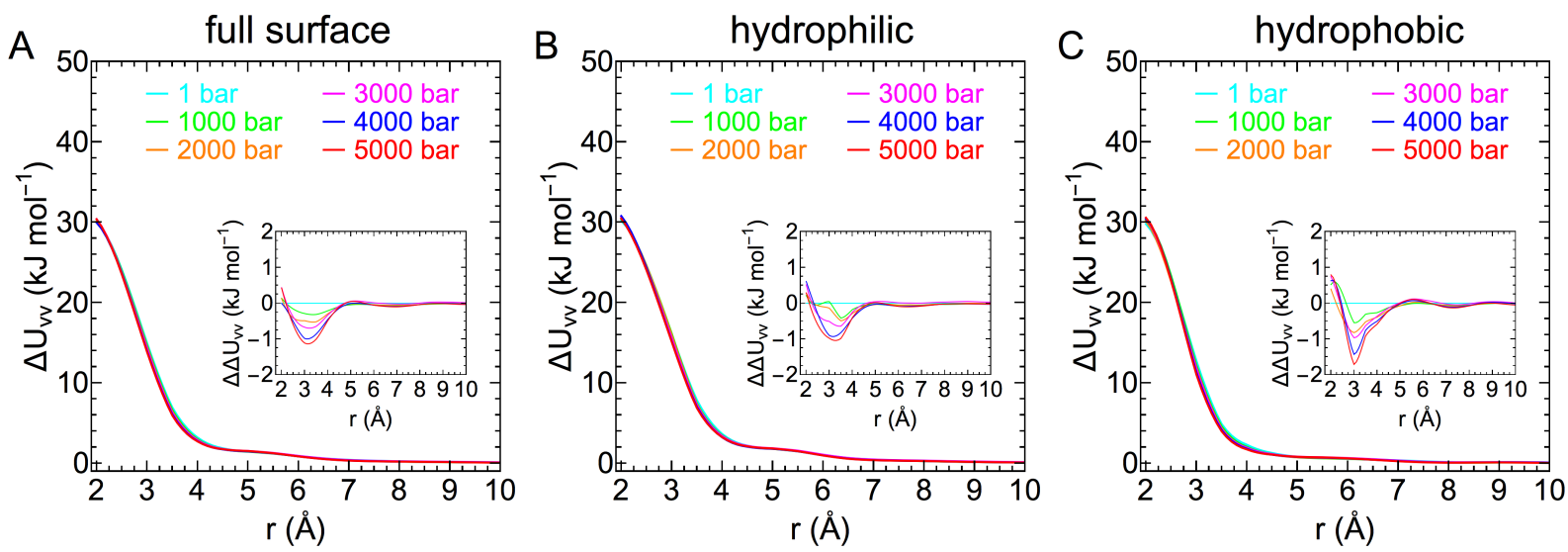

Figure S2: Water-water interaction energies per molecule, $\Delta U_{\mathrm{vv}}$, at different pressures, as a function of distance from the (A) full protein surface; (B) hydrophilic and (C) hydrophobic side-chains. Deviations of the corresponding water-water interaction energies from 1 bar are shown as insets in each panel.

\section{Spatially resolved dynamic and structural properties of}

\section{water}

Using Green-Kubo's formula for diffusion, it can be shown that the diffusion coefficient of a water molecule can be computed from the time integral of the (center of mass) velocity time 
autocorrelation function, which is directly proportional to the zero frequency response of its corresponding VDoS

$$
D=\frac{\operatorname{VDoS}(0) k_{B} T}{12 m_{\mathrm{w}}}
$$

Here, $k_{B}$ is Boltzmann's constant, $T$ is the temperature, and $m_{\mathrm{w}}$ is the mass of a water molecule.

Therefore, the grid-based analysis of the VDoS for water molecules (i.e., their centers of mass), performed within the framework of the 3D-2PT solvent entropy expressions, also provides a local measure for short time diffusion in the observation time window of $1.6 \mathrm{ps}$ and water translational mobility.

The three-dimensional grid used for the 3D-2PT analysis, is also used to compute a spatially resolved tetrahedral order parameter for water molecules, $q(\mathbf{r})$. The latter is defined as introduced by Errington et al. ${ }^{1}$ and takes into account cosines of the angles formed by the oxygen coordinates of a water molecule and its four nearest coordinating neighbors (HB donor or acceptor from another water molecule or the protein).

$$
q=1-\frac{3}{8} \sum_{i=1}^{3} \sum_{j=i+1}^{4}\left(\cos \psi_{i j}+\frac{1}{3}\right)^{2} .
$$

Here, $\psi_{i j}$ is the angle made by the central molecule with its nearest $i^{\text {th }}$ and $j^{\text {th }}$ neighbors. The theoretical output values for $q(\mathbf{r})$ range from -3 to 1 . However, in most environments values between 0 (ideal gas) and 1 (perfectly tetrahedral environment) are expected. 

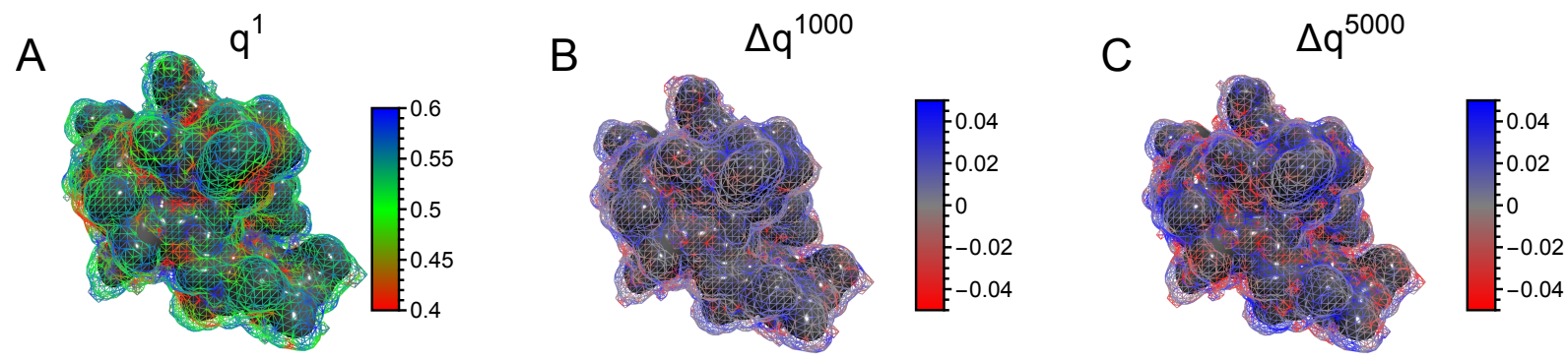

Figure S3: Three-dimensional representation of properties of water molecules in the protein hydration shell at $3 \AA$ from the protein surface: (A) tetrahedral order parameter at 1 bar; and $(B)-(C)$ deviations of tetrahedral order parameter at the corresponding pressures from 1 bar.
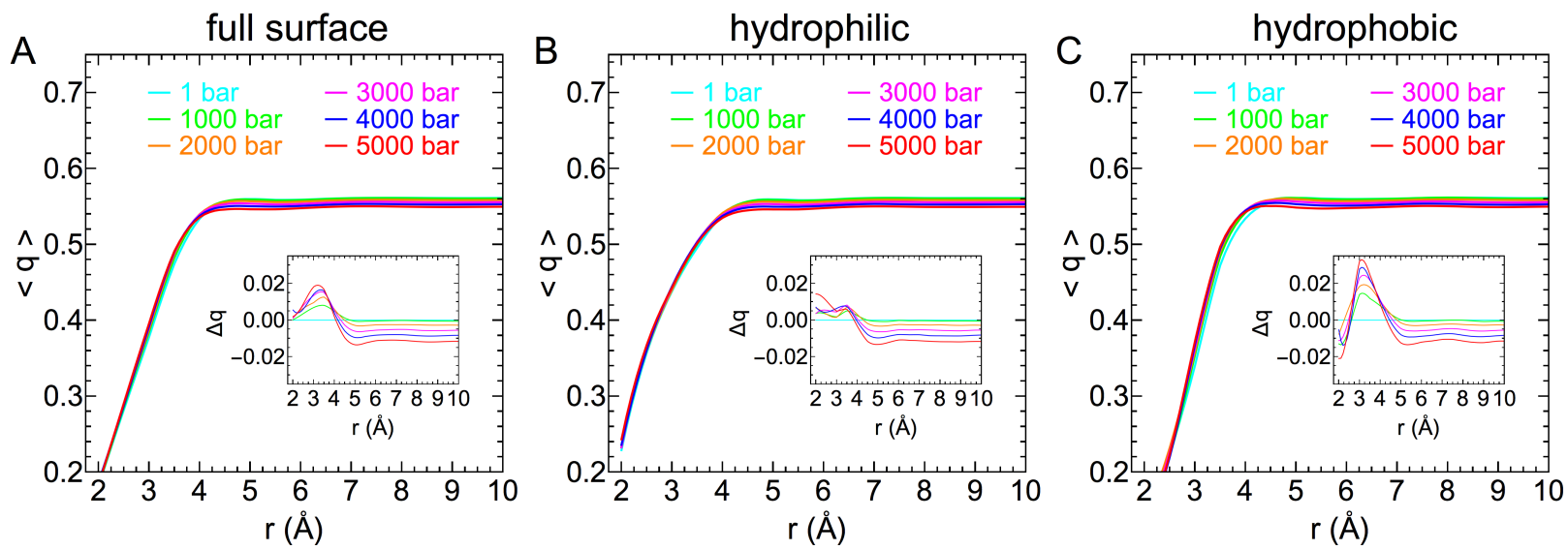

Figure S4: Water order parameter as a function of distance from the (A) full protein surface; (B) hydrophilic and (C) hydrophobic side-chains at the corresponding pressures (1-5000 bar). Deviations of the corresponding order parameter from 1 bar are shown as insets in each panel. 

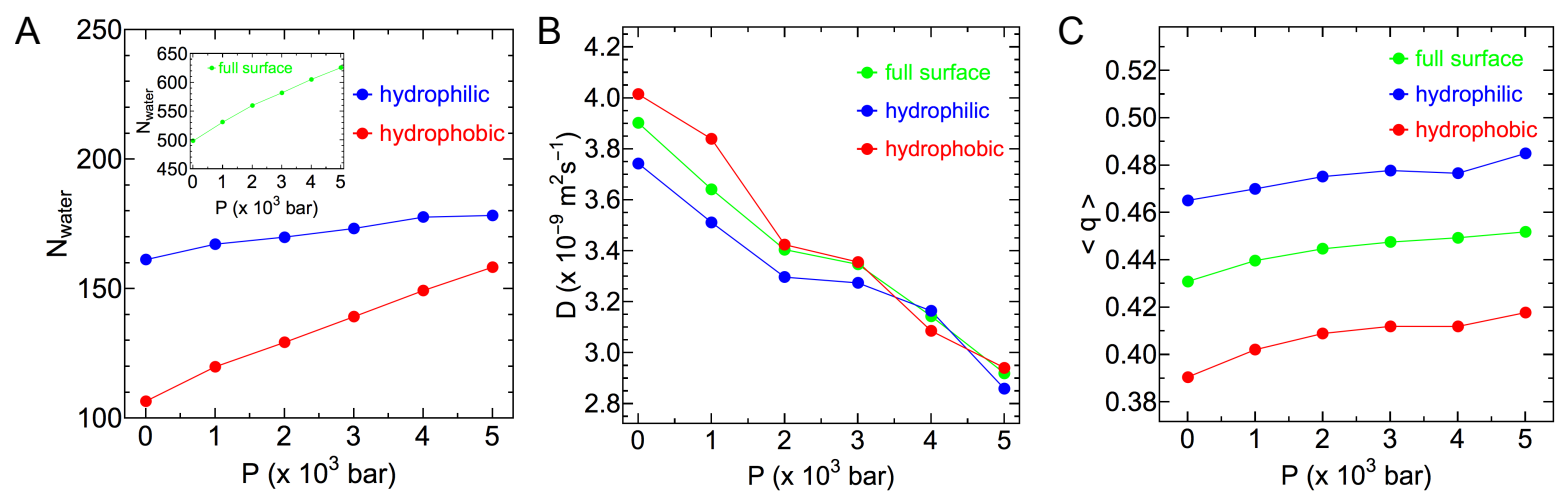

Figure S5: Structural and dynamic properties of hydration water molecules as a function of pressure. Shown are the results for water molecules within the first hydration shell of full protein surface (green), hydrophilic surface (blue), and hydrophobic surface (red). (A) numbers of hydration water molecules; (B) averaged water diffusion coefficients; and (C) averaged water tetrahedral order parameters. 


\section{References}

(1) Errington, J. R.; Debenedetti, P. G. Relationship between structural order and the anomalies of liquid water. Nature 2001, 409, 318. 